\title{
Conservative, minimally invasive and open surgical repair for management of acute ruptures of the Achilles tendon: a clinical and functional retrospective study
}

\author{
Gayle Maffulli ${ }^{1}$ \\ Angelo Del Buono2 \\ Paula Richards ${ }^{3}$ \\ Francesco Oliva ${ }^{4}$ \\ Nicola Maffulli ${ }^{1,5}$
}

1 Centre for Sports and Exercise Medicine, Barts and The London School of Medicine and Dentistry, Mile End Hospital, London, UK

2 Department of Orthopaedic and Trauma Surgery, Campus Biomedico University of Rome, Rome, Italy

3 Department of Radiology, University Hospitals of North Midlands NHS Trust, Stoke on Trent, UK

4 Department of Trauma and Orthopaedics, "Tor Vergata" University, Rome, Italy

5 Department of Musculoskeletal Disorders, University of Salerno, School of Medicine, Salerno, Italy

Corresponding author:

Francesco Oliva

Department of Trauma and Orthopaedics,

"Tor Vergata" University

Viale Oxford 81

00133 Rome, Italy

E-mail: olivafrancesco@hotmail.com

\section{Summary}

Background: At present, it is unclear which is the best management for Achilles tendon rupture.

Purpose: We assess the clinical, functional and imaging outcomes of active patients undergoing 3 different types of management for acute subcutaneous rupture of the Achilles tendon, including conservative cast immobilization, traditional open surgery and percutaneous repair.

Methods: 26 active patients were managed for a rupture of the Achilles Tendon from January 2007 to March 2008. Anthropometric measurements, Functional assessment, Isometric strength, Ultrasonographic assessment, Patient satisfaction, Working life, Physical activity, Functional score and Complications were recorded retrospectively. Results: All 23 (21 men, 2 women) patients were reviewed at a minimum follow-up of 24 months (average 25.7, range 24 to 32 months, SD: 6.3) from the index injury. Thermann scores and patient satisfaction were significantly higher following surgery than conservative management with no significance between open and minimally invasive operated patients. Sensitive disturbances occur in up to $12 \%$ of open repairs and $1.8 \%$ of patients managed nonsurgically.

Conclusions: Clinical and functional outcomes following surgical repair, percutaneous and open, of the Achilles tendon are significantly improved than following conservative management. Level of evidence: Level III.

KEY WORDS: Achilles tendon, tendon repair, Achilles tendon ruptures, Achilles tendon repair, miniinvasive procedures.

\section{Introduction}

The tendo Achillis is the thickest and strongest tendon of the human body ${ }^{1,2}$, frequently injured and ruptured in running and jumping activities, especially in men aged 30 to 40 years $^{3}$. At present, it is unclear which is the best management ${ }^{4-6}$ : if conservative and 'classical' open management are comparable for functional results ${ }^{7}$, surgery may produce lower re-rupture rate, allow early functional treatment, less calf atrophy, and stronger push off, at the expenses of long incisions, wound complications such as infections, and, occasionally, painful scars ${ }^{4}$.

Minimally invasive repair techniques are increasingly allowing to decrease traditional postoperative complications $^{8-10}$, permit accurate opposition of the tendon ends, allow improved cosmesis, and prevent wound breakdown ${ }^{11-13}$, but may damage to the sural nerve ${ }^{14}$. Percutaneous techniques, comparable for clinical effectiveness ${ }^{15}$ to traditional open procedures, are advocated for the lower complication rates ${ }^{16}$, and provide encouraging results in older individuals ${ }^{17}$, diabetic patients ${ }^{18}$, and high performance athletes ${ }^{19}$.

In this retrospective study, we present the clinical, functional and imaging outcomes of 26 active patients undergoing 3 different types of management for acute subcutaneous rupture of the Achilles tendon, including percutaneous repair, traditional open surgery, and conservative cast immobilization. 
Conservative, minimally invasive and open surgical repair for management of acute ruptures of the Achilles tendon: a clinical and functional retrospective study

\section{Materials and methods}

\section{Patient population}

The present investigation is a retrospective analysis of clinical, functional and imaging data on 26 active patients who presented to our institution to be managed for a rupture of the tendo Achillis. All these patients were treated from January 2007 to March 2008. Conservative management by cast immobilization, open surgery and minimally invasive percutaneous repair were undertaken in 8 (Group 1), 11 (Group 2) and 7 (Group 3 ) patients respectively. All the patients gave written informed consent and the local ethics committee approved all the procedures described.

\section{Clinical assessment}

The patients were assessed at baseline immediately before the index operation, and post-operatively at a minimum 2-year follow-up. Age, sex, side injured, mode of injury, pre-operative sport activities were recorded (Tabs. I, II). The diagnosis of rupture was performed clinically by the senior Author (NM): Simmond's ${ }^{20}$ and Matles's ${ }^{21}$ test, tenderness, and site of pain were assessed in all patients. After treatment, symptoms of pain, tenderness, stiffness, footwear limitations were recorded.

Table I. Preoperative demographics and clinical assessments.

\begin{tabular}{ll}
\hline Male/Female ratio & $15 / 11$ \\
Mean height $(\mathrm{cm})$ & $172.4(160-198)$ \\
Mean weight $(\mathrm{Kg})$ & $78.1(57-105)$ \\
Left/Right & $15 / 11$ \\
Average follow-up & $25.7(25-32)$ months \\
Mean age at surgery & 48.9 years (30-65; SD: 13.4)
\end{tabular}

Table II. Injury modalities.

\begin{tabular}{ll}
\hline Activity & N of patients \\
\hline Rugby & 5 \\
Running & 5 \\
Basket & 3 \\
Tennis & 3 \\
Soccer & 2 \\
Squash & 1 \\
Skiing & 1 \\
Cycling & 1 \\
Accident at home & 1 \\
Accident at work & 1 \\
\hline
\end{tabular}

\section{Ultrasound}

Ultrasonographic findings confirmed the diagnosis of tendon rupture in all patients. All US investigations were performed by a single experienced musculoskeletal radiologist unaware of the treatment. High-resolution 3-12 MHz linear transducers (Voluson-i, GE Medical Systems, UK) and Achilles tendon setting were used to optimize ultrasound assessments. With the subject prone, the heels overhanging couch, and the ankles flexed to $90^{\circ}$, both (normal and affected) the Achilles tendons were entirely assessed in the longitudinal plane. To avoid potential artifacts, the transducer was positioned perpendicular to the tendon fibers. The Achilles tendon thickness, measured in centimeters, was entirely assessed along its length. To exactly repeat the measures, measurement were carried out on longitudinal scans, at the musculotendinous junction (MTJ), the calcaneal insertion site $(\mathrm{Cl})$, and at a midtendon point. Tendon abnormalities were classified according to the grey-scale grading system. To assess tendon vascularity, Power Doppler (PD) settings were standardised with a gain of $68 \mathrm{~dB}$, sensitivity of 8 $\mathrm{cm} / \mathrm{s}$, and pulse repetition frequency of $800-1000$ $\mathrm{Hz}$. On longitudinal scans, tendon abnormalities were classified as focal thickening, hypoechoic areas, paratenon blurring and neovascularization.

\section{Open surgery}

Eleven patients (Group 2) were operated on using a previously described open technique under general anaesthesia using a thigh tourniquet inflated to 300 $\mathrm{mmHg}^{37}$. An end-to-end repair was performed with a single modified Kessler suture, extending just proximal to the Achilles tendon insertion distally and just distal to the musculotendinous junction proximally. The suture material used was either No. 1 Vicryl (Polyglactin 910 braided absorbable suture, Ethicon, Johnson \& Johnson, European Logistics Centre, Brussels, Belgium) (7 patients) or No. 1 polydioxanone (Ethicon W9234T) (4 patients). The repair was tensioned to reproduce the physiological equinus present in the opposite ankle. A running circumferential suture with 3-0 Vicryl reinforced the core suture $^{19}$. The repair was thicker than the original tendon, and the paratenon, even when viable, could not always be sutured over it. Continuous 4-0 Vicryl reabsorbable sutures were used for the subcutaneous fat, and the skin was closed with interrupted 4.0 Ethilon (Ethicon, Johnson \& Johnson) (5 patients) or with a subcuticular 4-0 Vicryl reabsorbable suture (6 patients). The skin wound was dressed with gauze, and sterile plaster wool was applied, followed by a below-the-knee plaster of Paris cast without increasing the natural minimal equinus of the ankle. When the cast had dried, patients were encouraged to mobilize with the use of crutches, under the direction of a physical therapist. Patients were discharged the day after the operation. 


\section{Percutaneous repair}

All surgical procedures were performed by a single experienced board certified fellowship trained orthopaedic surgeon (NM) under local anaesthesia by injection. With the patient prone, and no tourniquet, skin preparation is performed in the usual fashion, and sterile drapes are applied. The percutaneous repair was performed according to the technique described by Carmont and Maffulli22.

Briefly, the palpable tendon defect is marked.

A $1 \mathrm{~cm}$ transverse incision is made over the defect using a size 11 blade. Four longitudinal stab incisions are made lateral and medial to the tendon $6 \mathrm{~cm}$ proxi$\mathrm{mal}$ to the palpable defect. Two further longitudinal incisions on either side of the tendon are made 4-6 $\mathrm{cm}$ distal to the palpable defect. Forceps are then used to mobilise the tendon from beneath the subcutaneous tissues.

A $9 \mathrm{~cm}$ Mayo needle (BL059N, \#B00 round point spring eye, B Braun, Aesculap, Tuttlingen, Germany) is threaded with 2 double loops of Number 1 Maxon (Tyco Healthcare, Norwalk, CT). A Bunnel type of configuration is used for the proximal stump, and, after all the throws have been competed the suture is tested for security by pulling with both ends of the Maxon in a proximal to distal direction, through the transverse incision.

Another double loop of Maxon is then passed between the distal stabs incisions through the tendon, and in turn through the tendon and out of the transverse incision starting distal to the transverse passage in a half Kessler fashion. The ankle is held in full plantar flexion, and in turn opposing ends of the Maxon thread are tied together with a double throw knot, and then three further throws before being buried using the forceps. A clip is used to hold the first throw of the lateral side to maintain the tension of the suture.

A subcuticular Biosyn suture 3.0 (Tyco Healthcare, Norwalk, CT) is used to close the transverse incision, and Steri-strips (3M Health Care, St Paul, MN) are applied to the stab incisions. Finally, a Mepore dressing (Molnlycke Health Care, Gothenburg, Sweden) is applied, and a bivalved removable scotch cast in full plantar flexion is applied being held in place with Velcro straps.

\section{Post-operative management and follow-up}

Group 2 and Group 3. The post-operative management was in line with what previously described ${ }^{23}$ Briefly, patients were allowed home on the day of surgery, and allowed to weight bear as able on the metatarsal heads of the operated leg using elbow crutches. At the time of discharge from the hospital after the operation, all patients were given an appointment for review 2 weeks postoperatively. At 2 weeks, the plaster was removed, and a commercially available removable walker was applied, with five wedges, each $1.2 \mathrm{~cm}$ thick, at the heel. Proprioception, active plantar flexion, inversion and eversion exercises were allowed against manual resistance provided by a physiotherapist. One heel wedge was removed every other week, and at 6 weeks postoperatively the patients were left free of the cast and re- ferred to physiotherapy for active mobilization. At 10 or 12 weeks postoperatively, patients were assessed as to whether they were able to undertake more vigorous physiotherapy. Further follow-ups at 14 and 18 weeks were arranged. Patients were reviewed during the $6^{\text {th }}$ post-operative month. They were then followed up at 3 months intervals and discharged 12 months after the operation, once they were able to perform at least five toe raises unaided on the operated leg and after they returned to their work or sport.

\section{Clinical assessment}

For the purposes of the present study, patients were assessed at an average of 25.7 months (range 24 to 32 months, SD: 6.3) from the operation. At the last assessment, the modified Thermann total score was used to assess post-operative symptoms and physical activity outcome. Clinical examination and functional evaluation were performed by a single trained examiner not involved in the surgery. Patients were asked to classify their satisfaction into a 4 grade system varying from very satisfied (1) to dissatisfied (4), and the return to sport activity as complete resumption, minimal loss of activity, diminished level of participation, and limited participation. The patients were also examined for skin puckering, sensation abnormalities, stiffness, weakness, footwear limitations and sensation to the weather.

A fully trained anthropometrist able to reproduce within $5 \%$ the duplicate measurements on the same calf ${ }^{24}$ measured the maximum calf circumference in both the affected and the contralateral leg 10 centimetres below the tibial tuberosity, by using a commercially available steel tape measurer ${ }^{25}$.

Isometric plantar flexion strength of the gastrocsoleus muscle complex was measured bilaterally with the ankle in neutral $\left(0^{\circ}\right)$ in the fashion described in other reports $^{23}$. Each patient was allowed three maximal isometric voluntary contractions, and the highest was used for the purposed of this report. The apparatus was calibrated before and after each patient using the protocol previously described ${ }^{23}$. Dorsal and plantar flexions were measured using a goniometer.

\section{Statistical analysis}

This was carried out using SPSS software v.14.0 (SPSS Inc., Chicago, Illinois) using independent sample t-tests for assessing differences between groups. Muscle function parameters and range of movement of the ankle were calculated as a percentage of the normal side, and Student's t-test was used to compare the groups. Linear regression analysis was used to calculate correlation between imaging findings, clinical and functional outcomes.

\section{Results}

All 23 (21 men, 2 women) patients were reviewed at a minimum follow-up of 24 months (average 25.7, range 24 to 32 months, SD: 6.3 ) from the index operation. 
Conservative, minimally invasive and open surgical repair for management of acute ruptures of the Achilles tendon: a clinical and functional retrospective study

\section{Anthropometric measurements, functional assessment, and isometric strength}

The maximum calf circumference was significantly decreased in the operated limb of patients who had undergone open and percutaneous repair, with greater circumference in group 1 patients $(41.3 \pm 5.0$ $\mathrm{cm}$, versus $35.4 \pm 4.5$ in Group 2 and 3, not significant). The maximum calf circumference of the operated leg, $36.6 \pm 4.4 \mathrm{~cm}$, was significantly lower than that $(39.2 \pm 4.3 \mathrm{~cm})$ of the uninjured side $(p<.0001)$. The average isometric strength of $49.5 \pm 17.7 \mathrm{~N}$ on the operated limb was significantly decreased $(P=0.002)$ compared to the average value of $61.7 \pm 20.1 \mathrm{~N}$ on the uninjured side. Although a greater proportion of patients in Group 1 had excellent or good results, the difference was not statistically significant and there were remarkably few poor results in either group. The t-test paired sampled statistics showed that the operated limb was less strong than the non-operated one after both open $(P=0.01)$ and percutaneous surgery $(P=0.05)$. There was no statistically significant difference on the operated side between the patients, regardless of the management; however, the isometric strength on the operated side was higher in Group 1 patients.

\section{Ultrasonographic assessment}

The average anteroposterior diameter of the ruptured tendon was $11.7 \mathrm{~mm}$ (SD, 3.3) in Group 1, 12.1 (SD, 3.4) in Group 2, and 11.0 (SD, 1.8) in Group 3 patients, with no difference in the ruptured tendons regardless of the method of postoperative management. The patients' contralateral tendon was significantly thinner in Group $1(\mathrm{P}=0.008)$ and $2(\mathrm{P}<$ 0.00001 ) than in Group 3 patients. The patients' contralateral tendon was significantly thinner in Group 1 $(P=0.008)$ and $2(P<0.00001)$. The average mediolateral diameter of the ruptured tendon was $20.4 \mathrm{~mm}$ (SD, 3.8) in Group 1, 18.5 (SD, 5.3) in Group 2, and 20.9 (SD, 2.2) in Group 3 patients, with no difference in the ruptured tendons regardless of the method of postoperative management. The contra-lateral healthy tendon was thinner, not significantly, in all the 3 groups. Hypo-echoic and hyper-echoic areas were found in the ruptured tendon of 13 (5 in group 1, 5 in Group 2 and 3 in Group 3) and 3 patients (all in Group 2), respectively. One patient (in Group 2) showed areas of hypoechogenicity in the un-ruptured contralateral tendon.

\section{Patient satisfaction, working life and physical ac- tivity}

Eighten patients (2 in Group 1, 9 in Group 2, and 7 in Group 3) were very satisfied with the results of surgery. Patients undergoing surgery were markedly more satisfied than those managed conservatively, with no inter-group difference following open and minimally invasive AT repair $(p=0.88)$. There was no correlation between patient satisfaction and AP $(P=0.96)$ and $\mathrm{ML}(\mathrm{P}=0.56)$ diameters, and US echoic findings $(P=0.79)$ of the tendon.

Eighteen patients were employed at the time of their injury, and 8 were retired. By 12 months after the index injury, all patients employed at the time of their Achilles tendon rupture had returned to work, and no patient changed jobs after their Achilles tendon rupture. Patients returned to their activities after an average period of 12 weeks from the rupture, without significant intergroup differences. The 3 patients ( 1 for each group) with a manual job returned to work at an average of 21.3 weeks. No significant intergroup differences were found in terms of time of return to working activities.

All the 26 patients took part in regular physical activity, and $23(90 \%)$ were engaged in sports. Most patients had returned to regular physical activity by 12 months after the index injury. There were no significant differences in the rate of return to regular physical activity between the 3 groups. However, the average time to return to sport was 35.3 months (range, 26 to 52) in Group 1 patients, 28 weeks (range, 10 to 52) in Group 2 patients, and 29 in Group 3 patients (range, 12 to 52), with no statistically significant intergroup differences.

\section{Functional score}

The average modified Thermann total score for all subjects was 69.6, ranging from 38 to 91 , with significant higher scores in Group 2 and 3 patients than in Group 1 those. There was no statistically significant association between the VISA-A score and patient satisfaction. The scores were not significantly associated with AP $(p=0.80)$ and $L L(p=0.22)$ diameters and echoic $(p=0.34)$ findings, and age at injury $(p=0.56)$.

At the latest follow-up, of the $23(90 \%)$ patients participating in sports activities, $16(80 \%)$ had returned to the pre-operative activity, 2 changed to lower activity, and 1 increased performance to higher activity (Tab. III). Three patients gave up sport. Dorsi-flexion averaged $35.2^{\circ}$ (range, $5-30^{\circ}$ ) on the operated ankle and $31.9^{\circ}$ (range, $5-30^{\circ}$ ) on the contra-lateral, with no significant inter-side difference $(p=0.06)$. Comparing average plantar-flexions to the operated $\left(43.4^{\circ}\right)$ and contra-lateral $\left(43.3^{\circ}\right)$ side, we found no significant inter-side difference, $p=0.95$.

\section{Complications}

No patient experienced any superficial infection of the surgical wounds. Four patients reported hyperesthesia over the lateral aspect of the leg, and 6 supramalleolar dysesthesia at the first post-operative outpatient assessment, 9 patients ( 6 in the open and 3 in the percutaneous group) referred skin puckering. All neurological symptoms resolved spontaneously within three months after treatment surgery. 


\section{Discussion}

Management of acute ruptures of the tendo Achillis ${ }^{4}$ is varied and controversial, with no significant clearly proven advantages comparing outcomes following available procedures. Surgical repair is advocated for active patients who demand early return to high functional status, but the evidence is that surgery is cost$l y$, and produces higher complication rates than conservative management ${ }^{26}$. Conservative management, especially when followed by an accelerated rehabilitation program, is acceptable for reported clinical outcomes and avoidance of soft-tissue complications, relatively common after open surgery, but it may be more susceptible to the occurrence of re-ruptures ${ }^{27}$, up to $13 \%$, probably resulting from an incomplete healing of the gap between the two tendon stumps ${ }^{28}$, and to the development of muscle strength deficits, generally secondarily to the long time period the tendon is in lengthened position ${ }^{29}$.

This is a minimum 2-year follow-up retrospective analysis of 26 patients undergoing 3 different modalities of management for acute AT rupture. We found that percutaneous repair is a good compromise, which allow accurate re-approximation of the tendon ends, and avoid wound healing complications ${ }^{30}$. Although minimally invasive procedures better preserve the vascularization of the peritenon and its gliding surface ${ }^{31}$, some concerns arise from the intra-operative difficulty to visualize the exact position and juxtaposition of the torn tendon ends ${ }^{26,30}$. In our hands, it is possible to palpate the gap and properly locate the position of the ends 22 . In the present investigation, the post-operative Thermann scores and patient satisfaction were significantly higher following surgery than conservative management with no significance between open and minimally invasive operated patients. Sensitive disturbances occur in up to $12 \%$ of open repairs and $1.8 \%$ of patients managed nonsurgically ${ }^{32}$; in the present series, neurological complications were more frequent after open than minimally invasive repair. The percutaneous technique used in the present investigation reduces the risk for sural nerve damage, a troublesome complication in 0 to $60 \%$ of patients following percutaneous repair ${ }^{33}$, and impairing functional outcome ${ }^{14}$. Making longitudinal stab skin incisions parallel to the course of the nerve, as close to the lateral tendon edge as possible, prevents sural nerve injury ${ }^{30,33}$. Therefore, when sural nerve injury does occur, it would more likely be a longitudinal neurotomy rather than a transverse axonotmesis or neurotmesis, allowing the nerve to regenerate along its sheath, and minimize neuroma formation ${ }^{22}$. However, all neurological disturbances resolved spontaneously within 3 months. In open surgery, it was not possible to assess intra-operatively the status of the sural nerve. When performing a percutaneous repair, we undertake the procedure under local anaesthesia by direct skin infiltration to monitor the sural nerve during the operation with the possibility to retract the suture and start again the procedure if signs or symptoms of sural nerve entrapment appear.
After rupture, when the triceps surae is retracted, although a percutaneous procedure could be compromised for the inadequate juxtaposition of the torn ends, mostly if the ends are degenerated and uneven for length, our procedure allows the torn ends to be advanced distally symmetrically, simultaneously pulling the medial and lateral end of the suture, approximating the torn ends until the defect is no longer palpable, maintaining the foot in plantar flexion.

Although remarkably low re-rupture rates, ranging from 1 to $6.4 \%$ have been reported following surgery ${ }^{11}$, none of our patients experienced a re-rupture or required further surgery, probably in relationship with the regimen of immobilization in plantar flexion of the foot, the immediate weight bearing for the first two postoperative weeks, and the mobilization (active full plantar, inversion and eversion) exercises of the ankle and foot, aiming to prevent musculoskeletal changes associated with immobilization ${ }^{23}$.

As already shown, we did not find any differences in strength of the gastrosoleus complex, between open and percutaneous repair ${ }^{34}$; other studies have shown that the impact heel strike and toe off tests were significantly improved in patients undergoing percutaneous repair, probably in relationship to minimized pullout forces at the junction of the suture-tendon interface when performing this procedure ${ }^{30}$. Although the isometric strength of the gastrosoleus complex was statistically significantly lower than contra-laterally $(83.0 \%$ of the opposite side), we doubt that this decrease in strength interferes with the daily or sport activities. Importantly, dorsi-flexion and plantar flexion, although higher following open surgery, were not significantly different between the groups.

As reported in Table III, significantly higher Therman scores were evident following surgery than conservative treatment, with no significance between open and minimally invasive repair. Concerning the US assessment, we found that tendon diameters and echoic patterns are not associated with clinical, functional and satisfactory outcomes. Conversely, satisfaction and function are significantly increased after surgical repair, whether open or percutaneous, of the Achilles tendon. We are aware that limitations of our study are the relatively small sample size and the relatively short follow-up and that the evidence given is not as strong as that produced by a randomized controlled trial.

A strength of the present investigation is that we were able to follow-up the whole of our initial cohort originally operated with this technique, but we acknowledge that the present study is just a comparative study, with no attempts made at randomization.

\section{Conclusion}

Clinical and functional outcomes following surgical repair, percutaneous and open, of the Achilles tendon are significantly improved than following conservative management, but larger investigation using more robust study design with longer follow-up and more ex- 
Conservative, minimally invasive and open surgical repair for management of acute ruptures of the Achilles tendon: a clinical and functional retrospective study

Table III. Sport activity and Achilles tendon rupture score.

\begin{tabular}{|c|c|c|c|c|c|}
\hline Patient & Sport activity & Preoperative level & Postoperative level & ATRS score & Age at injury \\
\hline 1 & Rugby & Club & Recreational & 60 & 54 \\
\hline 2 & Tennis & Recreational & Recreational & 65 & 65.2 \\
\hline 3 & Running & Recreational & Recreational & 95 & 65.5 \\
\hline 4 & Rugby & Recreational & Stop activity & 53 & 28.4 \\
\hline 5 & Soccer & Club & Club & 79 & 34.3 \\
\hline 6 & Running & Recreational & Recreational & 70 & 49.1 \\
\hline 7 & Basket & Club & Club & 96 & 31.5 \\
\hline 8 & Running & Recreational & Stop activity & 94 & 63.6 \\
\hline 9 & Tennis & Recreational & Recreational & 79 & 51.5 \\
\hline 10 & Skiing & Recreational & Recreational & 87 & 45.4 \\
\hline 11 & Running & Recreational & Recreational & 99 & 50.5 \\
\hline 12 & Running & Recreational & Recreational & 91 & 29.7 \\
\hline 13 & Running & Club & Club & 98 & 33.8 \\
\hline 14 & Rugby & Recreational & Recreational & 72 & 37.5 \\
\hline 15 & Tennis & Recreational & Recreational & 97 & 40.3 \\
\hline 16 & Basket & Recreational & Recreational & 95 & 36.7 \\
\hline 17 & Rugby & Recreational & Recreational & 70 & 43.5 \\
\hline 18 & Cycling & Recreational & Recreational & 92 & 32.6 \\
\hline 19 & Tennis & Recreational & Recreational & 64 & 36.9 \\
\hline 20 & Basket & Club & Recreational & 92 & 38.3 \\
\hline 21 & Squash & Recreational & Recreational & 99 & 38 \\
\hline 22 & Rugby & Recreational & Club & 99 & 36.1 \\
\hline 23 & Soccer & Recreational & Recreational & 93 & 26.7 \\
\hline
\end{tabular}

tensive, clinically relevant outcome measures are needed to confirm this.

\section{Conflict of interest}

The Author has no financial or personal relationships with other people or organizations that could inappropriately influence their work.

\section{Ethics approval}

All procedures described in this study were approved by the Ethics Committee of our Institution. All patients gave their informed consent in written form. Furthermore the manuscript follow the ethical to international standards according and as required by the MLTJ Journal ${ }^{35}$.

\section{Contributors}

All Authors made substantial contributions to the intellectual content of the manuscript and have all ap- proved the final version. SG, MD, NM and VD conceived the study. ADB, FO and NM performed the review of the literature and wrote the initial draft.

\section{References}

1. O'Brien M. Functional anatomy and physiology of tendons. Clin Sports Med. 1992;11:505-520.

2. Doral MN, Alam M, Bozkurt M, Turhan E, Atay OA, Donmez G, Maffulli N. Functional anatomy of the Achilles tendon. Knee Surg Sports Traumatol Arthrosc. 2010;18:638-643.

3. Maffulli N, Ajis A, Longo UG, Denaro V. Chronic rupture of tendo Achillis. Foot Ankle Clin. 2007;12:583-596.

4. Wong J, Barrass V, Maffulli N. Quantitative review of operative and nonoperative management of achilles tendon ruptures. Am J Sports Med. 2002;30:565-575.

5. Ebinesan AD, Sarai BS, Walley GD, Maffulli N. Conservative, open or percutaneous repair for acute rupture of the Achilles tendon. Disabil Rehabil. 2008:30:1721-1725.

6. Amlang MH, Maffuli N, Longo UG, Stubig T, Imrecke J, Hufner T. Surgical treatment of Achilles tendon rupture. Unfallchirurg. 2010;113:712-720.

7. Nilsson-Helander K, Silbernagel KG, Thomee R, et al. Acute Achilles tendon rupture: a randomized, controlled study comparing surgical and nonsurgical treatments using validated outcome measures. Am J Sports Med. 2010;38: 2186-2193. 
8. Maffulli N, Longo UG, Denaro V. Complications after surgery or nonoperative treatment for acute achilles tendon rupture. Clin J Sport Med. 2009;19: 441-442.

9. Doral MN, Bozkurt M, Turhan E, et al. Percutaneous suturing of the ruptured Achilles tendon with endoscopic control. Arch Orthop Trauma Surg. 2009;129:1093-1101.

10. Maffulli N, Longo UG, Oliva F, Ronga M, Denaro V. Minimally invasive surgery of the achilles tendon. Orthop Clin North Am. 2009;40:491-498.

11. Cretnik A, Kosanovic M, Smrkolj V. Percutaneous suturing of the ruptured Achilles tendon under local anesthesia. J Foot Ankle Surg. 2004;43:72-81.

12. McClelland $D$, Maffulli N. Percutaneous repair of ruptured Achilles tendon. J R Coll Surg Edinb. 2002;47:613-618.

13. Longo UG, Ronga M, Maffulli N. Acute ruptures of the achilles tendon. Sports Med Arthrosc. 2009;17:127-138.

14. Maes $R$, Copin $G$, Averous $C$. Is percutaneous repair of the Achilles tendon a safe technique? A study of 124 cases. Acta Orthop Belg. 2006;72:179-183.

15. Goren D, Ayalon M, Nyska M. Isokinetic strength and endurance after percutaneous and open surgical repair of Achilles tendon ruptures. Foot Ankle Int. 2005;26:286-290.

16. Khan RJ, Fick D, Keogh A, Crawford J, Brammar T, Parker M. Treatment of acute achilles tendon ruptures. A meta-analysis of randomized, controlled trials. J Bone Joint Surg Am. 2005; 87:2202-2210.

17. Maffulli N, Longo UG, Ronga M, Khanna A, Denaro V. Favorable outcome of percutaneous repair of achilles tendon ruptures in the elderly. Clin Orthop Relat Res. 2009;468:10391046.

18. Maffulli N, Longo UG, Maffulli GD, Khanna A, Denaro V. Achilles tendon ruptures in diabetic patients. Arch Orthop Trauma Surg. 2010;131:33-38.

19. Maffulli N, Longo UG, Maffulli GD, Khanna A, Denaro V. Achilles tendon Ruptures in Elite Athletes. Foot Ankle Int. 2011;32.

20. Simmonds FA. The diagnosis of the ruptured Achilles tendon. Practitioner. 1957; 179:56-58.

21. Matles AL. Rupture of the tendo Achilles. Another diagnostic sign. Bull Hosp Joint Dis. 1975;36:48-51.

22. Carmont MR, Maffulli N. Modified percutaneous repair of ruptured Achilles tendon. Knee Surg Sports Traumatol Arthrosc. 2008;16:199-203.
23. Maffulli N, Tallon C, Wong J, Lim KP, Bleakney R. Early weightbearing and ankle mobilization after open repair of acute midsubstance tears of the achilles tendon. Am J Sports Med. 2003;31:692-700.

24. Maffulli N, Kenward MG, Irwin AS, Porter RW. Assessment of late results of surgery in talipes equino-varus: a reliability study. Eur J Pediatr. 1997;156:317-319.

25. Haggmark T, Liedberg H, Eriksson E, Wredmark T. Calf muscle atrophy and muscle function after non-operative vs operative treatment of achilles tendon ruptures. Orthopedics. 1986;9:160-164.

26. Maffulli N. Rupture of the Achilles tendon. J Bone Joint Surg Am. 1999;81:1019-1036.

27. Willits $\mathrm{K}$, Amendola $\mathrm{A}$, Bryant $\mathrm{D}$, et al. Operative versus nonoperative treatment of acute Achilles tendon ruptures: a multicenter randomized trial using accelerated functional rehabilitation. J Bone Joint Surg Am. 2010;92:2767-2775.

28. Wills CA, Washburn S, Caiozzo V, Prietto CA. Achilles tendon rupture. A review of the literature comparing surgical versus nonsurgical treatment. Clin Orthop Relat Res. 1986;156-163.

29. Inglis AE, Scott WN, Sculco TP, Patterson AH. Ruptures of the tendo achillis. An objective assessment of surgical and nonsurgical treatment. J Bone Joint Surg Am. 1976;58:990-993.

30. Ma GW, Griffith TG. Percutaneous repair of acute closed ruptured achilles tendon: a new technique. Clin Orthop Relat Res. 1977:247-255.

31. Kosanovic M, Cretnik A, Batista M. Subcutaneous suturing of the ruptured Achilles tendon under local anaesthesia. Arch Orthop Trauma Surg. 1994;113:177-179.

32. Cetti R, Christensen SE, Ejsted R, Jensen NM, Jorgensen U. Operative versus nonoperative treatment of Achilles tendon rupture. A prospective randomized study and review of the literature. Am J Sports Med. 1993;21:791-799.

33. Majewski M, Rohrbach M, Czaja S, Ochsner P. Avoiding sural nerve injuries during percutaneous Achilles tendon repair. Am J Sports Med. 2006;34:793-798.

34. Bradley JP, Tibone JE. Percutaneous and open surgical repairs of Achilles tendon ruptures. A comparative study. Am J Sports Med. 1990;18:188-195.

35. Padulo J, Oliva F, Frizziero A, Maffulli N. Muscles, Ligaments and Tendons Journal - Basic principles and recommendations in clinical and field science research: 2016 Update. MLTJ. 2016; 6(1):1-5. 